\title{
Further signatures to support the tetraquark mixing framework for the two light-meson nonets
}

\author{
Hungchong Kim, ${ }^{1,2, *}$ K. S. Kim, ${ }^{3}$ Myung-Ki Cheoun, ${ }^{4}$ Daisuke Jido, ${ }^{5}$ and Makoto Oka ${ }^{6}$ \\ ${ }^{1}$ Research Institute of Basic Science, Korea Aerospace University, Goyang, 412-791, Korea \\ ${ }^{2}$ Center for Extreme Nuclear Matters, Korea University, Seoul 02841, Korea \\ ${ }^{3}$ School of Liberal Arts and Science, Korea Aerospace University, Goyang, 412-791, Korea \\ ${ }^{4}$ Department of Physics, Soongsil University, Seoul 156-743, Korea \\ ${ }^{5}$ Department of Physics, Tokyo Institute of Technology, Meguro, Tokyo 152-8551, Japan \\ ${ }^{6}$ Advanced Science Research Center, Japan Atomic Energy Agency, Tokai, Ibaraki 319-1195, Japan
}

(Received 29 October 2018; published 4 January 2019)

\begin{abstract}
In this work, we investigate additional signatures to support the tetraquark mixing framework that has been recently proposed as a possible structure for the two nonets, namely $a_{0}(980), K_{0}^{*}(800), f_{0}(500)$, and $f_{0}(980)$ in the light nonet and $a_{0}(1450), K_{0}^{*}(1430), f_{0}(1370)$, and $f_{0}(1500)$ in the heavy nonet. First, we advocate that the two nonets form the flavor nonet approximately satisfying the Gell-Mann-Okubo mass relation. Then we reexamine the mass ordering generated from the tetraquark nonets and show that this mass ordering is satisfied by the two nonets although the ordering in the heavy nonet is marginal. The marginal mass ordering however can be regarded as another signature for tetraquarks because it can be explained partially by the hyperfine masses calculated from the tetraquark mixing framework. The tetraquark mixing parameters are found to be independent of isospins giving additional support for the formation of the flavor nonets. In addition, we discuss the other approaches like two-quark pictures or meson-meson bound states, and their possible limitations in explaining the two nonets. As a peculiar signature distinguished from other approaches, we investigate the fall-apart coupling strengths into two vector mesons from our tetraquarks. Coupling strengths into the two-vector modes are found to enhance strongly in the heavy nonet while they are suppressed in the light nonet. The coupling ratios, which depend on the isospin channel, are found to be huge around $\sim 15$. This trend in the two-vector modes, which is opposite to that in the two-pseudoscalar fall-apart modes, can provide another testing ground for the tetraquark mixing framework. Some experimental evidence related to the phenomena is discussed particularly from the resonances belonging to the heavy nonet.
\end{abstract}

DOI: 10.1103/PhysRevD.99.014005

\section{INTRODUCTION}

For the last decade or so, an increasing number of exotic states reported from worldwide high energy facilities has triggered great excitement in the hadron community, especially because of the possibility that they might be the long-sought multiquark states. These exotic states include the pioneering resonance $X(3872)$ [1-4] measured in the $B$-meson decays and other resonances $X(3823)$, $X(3900), X(3940), X(4140), X(4274), X(4500)$, and $X(4700)$ [5-9] as well. Also the pentaquark candidates, $P_{c}(4380)$ and $P_{c}(4450)$, have been reported in Ref. [10]

\footnotetext{
*hungchong@kau.ac.kr
}

Published by the American Physical Society under the terms of the Creative Commons Attribution 4.0 International license. Further distribution of this work must maintain attribution to the author(s) and the published article's title, journal citation, and DOI. Funded by SCOAP ${ }^{3}$. from the $J / \psi p$ channel in the $\Lambda_{b}^{0} \rightarrow J / \psi K^{-} p$ decay. Recently, the authors of Ref. [11] reported the observation of $X(5568)$ from the D0 experiment at the Fermilab claiming that this could be a tetraquark with four different flavors because a molecular state composed of loosely bound $B_{d}$ and $K$ mesons is disfavored due to the large mass difference.

Theoretically, studies on tetraquarks in hadron spectroscopy are very diverse, ranging from the light mesons composed of $u, d, s$ quarks to the heavy mesons involving charm and bottom quarks. Even for the heavy mesons, the tetraquark investigation is further subdivided into various sectors such as hidden-charm [12-15], open-charm [16], doubly charmed [17-21] and triple [22] or fully charmed [23-26], and the similar states with bottom quarks. Eventually a unified approach for tetraquarks is anticipated because all the constituent quarks are bound by the color forces that are in principle independent on quark flavors. 
The most popular approach for tetraquarks is the diquark-antidiquark model [27-29] proposed long ago by Jaffe in his exploratory investigation of tetraquarks in the light mesons. In this approach, the tetraquarks are constructed by combining diquarks and antidiquarks. Since diquarks and antidiquarks are colored, the resulting tetraquarks can form tightly bound states by direct color forces. So the diquark is a sort of building block in constructing tetraquarks. A common practice is to use the spin-0 diquark with the color and flavor structures of $\left(\overline{\mathbf{3}}_{c}, \overline{\mathbf{3}}_{f}\right)$ because this diquark is most attractive among all the possible diquarks [30] if the binding is mainly driven by the color-spin interaction. Famous candidates are the light nonet consisting of $a_{0}(980), K_{0}^{*}(800), f_{0}(500)$, and $f_{0}(980)$ [27-35].

However, in its extension to the tetraquarks containing heavy quarks, the possible diquarks are not limited to the spin- 0 diquark and different diquarks are often adopted in the construction of tetraquarks. This is because of the possibility that the binding within a diquark can be provided by other mechanisms different from the color-spin interaction. For instance, the color-electric interaction can participate in holding the two quarks in a diquark [18]. Indeed, people are looking for bound states from doubly charmed ( $c c \bar{q} \bar{q}, q=u, d, s)$ [17-21]; triple ( $c c \bar{c} \bar{q})$ [22]; or fully charmed $(c c \bar{c} \bar{c})$ tetraquarks [23-26] in the diquarkantidiquark approach even though the heavy diquark, $c c$, cannot be the spin-0 diquark above. ${ }^{1}$ Actually, it is not clear whether the tetraquarks containing $c c$ can form a bound state or not $[18,19]$. For example, the authors of Ref. [24] suggested that $c c \bar{c} \bar{c}$ and $b b \bar{b} \bar{b}$ are unbound while the fully heavy tetraquark with different quark flavors, $b c \bar{b} \bar{c}$, is bound. The latter result can be understood if one assumes the dominance of the color-spin interaction because this interaction gives an attraction for the diquark with different quark flavors.

Even in the light meson system composed of $u, d, s$ quarks, one can introduce a different diquark, namely the spin-1 diquark with $\left(\mathbf{6}_{c}, \overline{\mathbf{3}}_{f}\right)$, and construct the second type of tetraquarks [36-38] in addition to the first type constructed from the most common spin-0 diquark discussed above. The spin-1 diquark can be used as a building block also because it forms a bound state even though it is less attractive than the spin-0 diquark above [30]. But the total binding, which is calculated by summing over pairwise interactions among all four quarks, is found to be more negative so the spin-1 diquark must be considered as an important ingredient in the formation of tetraquarks. The necessity of the spin-1 diquark is also supported by the QCD sum rule calculation for $D_{s}(2317)$ using diquarkantidiquark interpolating fields [39]. There, it is found that

\footnotetext{
${ }^{1}$ Since the diquark flavor, $c c$, is symmetric, its spin and color configurations are restricted either to $\left(J=1, \overline{\mathbf{3}}_{c}\right)$ or to $\left(J=0, \mathbf{6}_{c}\right)$. Both configurations are repulsive in color-spin interaction [30] and they are clearly different from the spin-0 diquark above.
}

the interpolating field containing the vector diquarks can describe $D_{s}(2317)$ equally well as the interpolating field containing the scalar diquarks.

The main aspect of Refs. [36-38] is that the two tetraquark types, one type constructed from the spin-0 diquark and the other type from the spin-1 diquark, mix strongly through the color-spin interaction. So the physical resonances can be identified by the eigenstates that diagonalize the hyperfine color-spin interaction. This tetraquark mixing framework is very promising as a possible structure for the two nonets in the review of the Particle Data Group (PDG) [40], $a_{0}(980), K_{0}^{*}(800), f_{0}(500)$, and $f_{0}(980)$ in the light nonet and $a_{0}(1450), K_{0}^{*}(1430), f_{0}(1370)$, and $f_{0}(1500)$ in the heavy nonet. Indeed, this structure has been tested relatively well in reproducing the mass splittings between the two nonets as well as the partial decay widths into two pseudoscalar mesons [36-38].

On the other hand, one can try other approaches for the two nonets in PDG. One immediate approach would be a two-quark picture with orbital angular momentum $\ell=1$. But, as we will discuss below, its simple applications do not explain the two nonets especially in achieving a phenomenological consistency with the mass ordering. Another approach is the meson-meson bound states separated with a long-range interaction. The hadronic molecular picture composed of $K \bar{K}$ or $\pi \eta$ has been proposed for $f_{0}(980)$ and $a_{0}(980)$ [41-44]. This molecular picture is also actively investigated in the heavy meson sectors including the recent exotic resonances [45-50]. As hybrid type approaches, there is a two-quark picture with hadronic intermediate states $[51,52]$ through the unitarized quark model. This approach has been extended to generate the physical states belonging to the two nonets dynamically from a single $q \bar{q}$ state in each isospin channel $[53,54]$. Other models also exist in the literature like the tetraquarks mixed with a glueball [55], the $P$-wave $q \bar{q}$ mixed with the four-quark $q q \bar{q} \bar{q}$ scalar nonet [56,57], or the tetraquarks including instantons [58]. Judging from various approaches, the current status on the nature of the two nonets is rather unclear and more studies are necessary in order to establish a realistic picture for the resonances being considered here. However, we believe that our tetraquark mixing framework provides a relatively simple picture and it may be worth pursuing further consequences of this model.

In this work, we investigate additional signatures to support the tetraquark mixing framework [36-38] as a plausible structure for the two nonets in PDG, the light and heavy nonets. First, we point out that the two nonets satisfy the Gell-Mann-Okubo mass relation approximately, which may indicate that the two nonets form the flavor nonets. Their tetraquark nature will be justified by demonstrating that their mass ordering is consistent with the tetraquark picture even though the ordering is marginal in the heavy nonet. We argue however that the marginal mass ordering can be regarded as more supporting evidence for our 
tetraquark mixing framework because it can be explained partially by the narrow splitting between the hyperfine masses calculated from the tetraquark mixing framework. Further evidence to support the flavor nonets can be seen from the fact that the mixing parameters in generating the heavy and light nonets are almost independent of the isospins. (See Sec. III C below.)

As comparative models, we examine the other approaches for the two nonets, namely the two-quark picture with orbital angular momentum $\ell=1$ and the meson-meson molecular picture. It will be pointed out that the two-quark picture can have only one configuration in the $J^{P}=0^{+}$channel, which is clearly not enough to accommodate the two nonets in PDG. Its application only to the heavy nonet is not realistic also due to the inconsistency in the mass ordering. We discuss the meson-meson picture as well and point out that this picture requires the additional multiplets to be found in PDG. One possible way to distinguish the tetraquark mixing framework from these pictures would be the fall-apart modes and their peculiar prediction from the light and heavy nonets.

Along this line, we study the fall-apart modes into the two-vector channels from our tetraquark system by recombining quarks and antiquarks in the wave functions. The color and spin factors as well as the flavor recombination factor will be calculated in detail. Because of the tetraquark mixing framework, the coupling strengths to the two vector mesons are found to be strongly enhanced for the heavy nonet but they are suppressed for the light nonet. This is in contrast to the fall-apart modes into the two pseudoscalar mesons whose coupling strengths are enhanced for the light nonet but suppressed for the heavy nonet $[37,38]$.

This paper is organized as follows. After a brief review on the tetraquark mixing framework in Sec. II, we give additional supporting arguments in Sec. III in identifying the two nonets as tetraquarks. In Sec. IV, we introduce other models such as two-quark picture or meson-meson molecule picture and discuss possible problems with them. We then present our formalism for the fall-apart modes of our tetraquarks into two vector mesons in Sec. V and propose the interesting phenomenological consequences in the fallapart strengths that differentiate between the light and heavy nonets. We summarize in Sec. VI.

\section{REVIEW ON TETRAQUARK MIXING FRAMEWORK}

To make our presentation self-contained, we begin with a brief review on the tetraquark mixing framework advocated in Refs. [36-38]. There, we have demonstrated that two tetraquark types are possible in the $J^{P}=0^{+}$channel within the diquark-antidiquark model. The first type is constructed by using the spin-0 diquark with color and flavor structures, $\left(\overline{\mathbf{3}}_{c}, \overline{\mathbf{3}}_{f}\right)$. The second type is constructed from the spin-1 diquark with $\left(\mathbf{6}_{c}, \overline{\mathbf{3}}_{f}\right)$. The spin and color configurations of the two tetraquark types are the following:

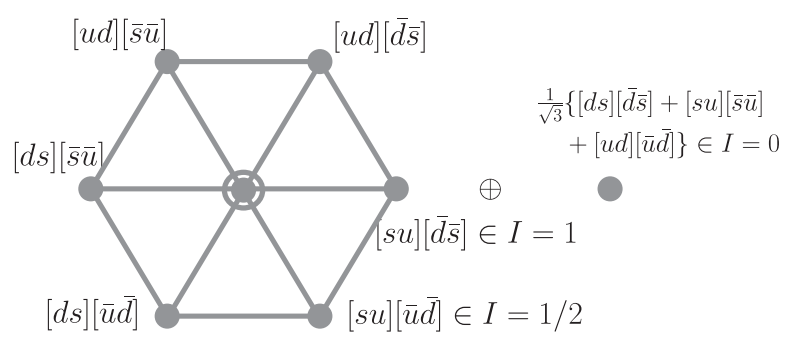

Two states at the center :

$$
\begin{aligned}
& \frac{1}{\sqrt{6}}\{[d s][\bar{d} \bar{s}]+[s u][\bar{s} \bar{u}]-2[u d][\bar{u} \bar{d}]\} \in I=0 \\
& \frac{1}{\sqrt{2}}\{[s u][\bar{s} \bar{u}]-[d s][\bar{d} \bar{s}]\} \in I=1
\end{aligned}
$$

FIG. 1. Weight diagram for the tetraquark nonet with explicit flavor wave functions. Here the bracket "[..]" denotes the antisymmetric combination, for example, $[s u]=\frac{1}{\sqrt{2}}(s u-u s)$. Note that the flavor structure is common for both tetraquark types, $|000\rangle$ and $|011\rangle$.

First type:

$$
\begin{gathered}
\left|J J_{12} J_{34}\right\rangle=|000\rangle, \\
\left|\mathbf{1}_{c} \overline{\mathbf{3}}_{c} \mathbf{3}_{c}\right\rangle=\frac{1}{\sqrt{12}} \varepsilon_{a b d} \varepsilon_{a e f}\left(q^{b} q^{d}\right)\left(\bar{q}^{e} \bar{q}^{f}\right),
\end{gathered}
$$

Second type:

$$
\begin{gathered}
\left|J J_{12} J_{34}\right\rangle=|011\rangle, \\
\left|\mathbf{1}_{c} \boldsymbol{6}_{c} \overline{\mathbf{6}}_{c}\right\rangle=\frac{1}{\sqrt{96}}\left(q^{a} q^{b}+q^{b} q^{a}\right)\left(\bar{q}^{a} \bar{q}^{b}+\bar{q}^{b} \bar{q}^{a}\right) .
\end{gathered}
$$

Here the state specifications are for the tetraquark, diquark, and antidiquark successively. In particular, $J$ denotes the tetraquark spin, $J_{12}$ the diquark spin, and $J_{34}$ the antidiquark spin. In our discussion below, the two tetraquark types are denoted mostly by their spin configurations, $|000\rangle$ and $|011\rangle$, unless explicit specification of the color configuration is necessary.

By construction, both tetraquarks have the same flavor structure; namely they form a nonet which can be broken down to an octet and a singlet $\left(\overline{\mathbf{3}}_{f} \otimes \mathbf{3}_{f}=\mathbf{8}_{f} \oplus \mathbf{1}_{f}\right)$. Figure 1 shows a weight diagram for the flavor nonet with explicit individual quark flavors. ${ }^{2}$ Other common features of the two tetraquarks are that the spin $(J)$, parity $(P)$, and charge conjugation $(C)$ are $J^{P C}=0^{++}$and their isospins are restricted to $I=0,1 / 2,1$.

Our claim in Refs. [36-38] is that the second tetraquark type must be considered along with the first tetraquark in the study of tetraquarks with $J^{P}=0^{+}$because they mix strongly through the color-spin interaction [60-63],

\footnotetext{
${ }^{2}$ One can easily construct this using a tensor notation. See Ref. [59] for technical details.
} 


$$
V_{\mathrm{CS}}=v_{0} \sum_{i<j} \lambda_{i} \cdot \lambda_{j} \frac{J_{i} \cdot J_{j}}{m_{i} m_{j}}
$$

Here $\lambda_{i}$ denotes the Gell-Mann matrix for $\mathrm{SU}(3)_{c}, J_{i}$ the spin, and $m_{i}$ the constituent quark mass. Because of this mixing, the hyperfine masses, which are the expectation values of the color-spin interaction with respect to $|000\rangle$ and $|011\rangle$, form a $2 \times 2$ matrix $^{3}$ for each isospin member. The upshot of this mixing is that physical resonances can be identified by the eigenstates that diagonalize this $2 \times 2$ matrix. Since the quark mass and the color-electric terms are already diagonal, almost proportional to the identity matrix in $|000\rangle$ and $|011\rangle$, the eigenstates of the hyperfine masses diagonalize the full Hamiltonian approximately.

This tetraquark mixing framework can be represented collectively by the formulas,

$$
\begin{gathered}
\mid \text { Heavy nonet }\rangle=-\alpha|000\rangle+\beta|011\rangle, \\
\mid \text { Light nonet }\rangle=\beta|000\rangle+\alpha|011\rangle,
\end{gathered}
$$

where the eigenstates, |Heavy nonet $\rangle$ and $\mid$ Light nonet $\rangle$, are identified by the two nonets in PDG. For the light nonet, we take the lowest-lying resonances in $J^{P}=0^{+}, a_{0}(980)$, $K_{0}^{*}(800), f_{0}(500), f_{0}(980)$. For the heavy nonet, we take the next higher resonances in $J^{P}=0^{+}, a_{0}(1450)$, $K_{0}^{*}(1430), f_{0}(1370), f_{0}(1500)$. These two nonets are separated by huge mass gaps, more than $500 \mathrm{MeV}$ or so, and they are also well separated from the other higher resonances in $J^{P}=0^{+}$. Their quantum numbers, experimental masses and widths are listed in Table I. Table II presents our results for the mixing parameters, $\alpha, \beta$, and the hyperfine masses calculated in the |Heavy nonet $\rangle$, $\mid$ Light nonet $\rangle$ bases [36,38].

This tetraquark mixing framework leads to interesting outcomes which can support the tetraquark structure of the two nonets [36-38]. Here we list some of the main results as the following.

(1) One surprising result is the inequality among the mixing parameters, $\alpha>\beta$. This implies that the light nonet members, $a_{0}(980), K_{0}^{*}(800), f_{0}(500)$, and $f_{0}(980)$, have more probability to stay in the configuration $|011\rangle$ rather than in $|000\rangle$. This result is originated from the fact that the second tetraquark, $|011\rangle$, is more compact than the first tetraquark, $|000\rangle$, namely $\left\langle 011\left|V_{\mathrm{CS}}\right| 011\right\rangle<\left\langle 000\left|V_{\mathrm{CS}}\right| 000\right\rangle$. This is very different from a common expectation that the light nonet is dominated by the configuration, $|000\rangle$. But this result is supported by the similar calculations [56] where this mixing was used only to

\footnotetext{
${ }^{3}$ To put this more precisely, one member belonging to $|000\rangle$ and the other with the same flavor member belonging to $|011\rangle$ participate in making a $2 \times 2$ matrix through the color-spin interaction.
}

TABLE I. Tetraquark candidates are listed here for the light and heavy nonets collected from PDG [40]. Note that the experimental masses are different from the numbers appearing in the meson nomenclatures.

\begin{tabular}{c|c|c|c|c|c}
\hline \hline & $J^{P C}$ & $I$ & Meson & Mass $(\mathrm{MeV})$ & $\Gamma(\mathrm{MeV})$ \\
\hline & $0^{++}$ & 1 & $a_{0}(980)$ & 980 & $50-100$ \\
Light nonet & $0^{+}$ & $1 / 2$ & $K_{0}^{*}(800)$ & 682 & 547 \\
& $0^{++}$ & 0 & $f_{0}(500)$ & $400-550$ & $400-700$ \\
& $0^{++}$ & 0 & $f_{0}(980)$ & 990 & $10-100$ \\
\hline & $0^{++}$ & 1 & $a_{0}(1450)$ & 1474 & 265 \\
Heavy nonet & $0^{+}$ & $1 / 2$ & $K_{0}^{*}(1430)$ & 1425 & 270 \\
& $0^{++}$ & 0 & $f_{0}(1370)$ & $1200-1500$ & $200-500$ \\
& $0^{++}$ & 0 & $f_{0}(1500)$ & 1505 & 109 \\
\hline \hline
\end{tabular}

TABLE II. Here are the hyperfine masses and the mixing parameters, $\alpha, \beta$, associated with the configuration mixing [Eqs. (6) and (7)] collected from Refs. [36,38]. For the isoscalar cases, we include the flavor fixing according to the "realistic case with fitting (RCF)." See Ref. [38] for details. Note that the hyperfine mass ordering, $\left.\left\langle V_{\mathrm{CS}}\right\rangle_{I=1}>\left\langle V_{\mathrm{CS}}\right\rangle_{I=1 / 2}\right\rangle\left\langle V_{\mathrm{CS}}\right\rangle_{I=0}\left(\mathbf{8}_{f}\right)$, is the same as the mass ordering in both nonets. Here $\left\langle V_{\mathrm{CS}}\right\rangle_{I=0}\left(\mathbf{8}_{f}\right)$ denotes the hyperfine mass of $f_{0}(500)$ or $f_{0}(1370)$ depending on the nonets.

\begin{tabular}{l|c|c|c|c|c}
\hline \hline Light nonet & $\left\langle V_{\mathrm{CS}}\right\rangle$ & Heavy nonet & $\left\langle V_{\mathrm{CS}}\right\rangle$ & $\alpha$ & $\beta$ \\
\hline$a_{0}(980)$ & -488.5 & $a_{0}(1450)$ & -16.8 & 0.8167 & 0.5770 \\
$K_{0}^{*}(800)$ & -592.7 & $K_{0}^{*}(1430)$ & -26.9 & 0.8130 & 0.5822 \\
$f_{0}(500)$ & -667.5 & $f_{0}(1370)$ & -29.2 & 0.8136 & 0.5814 \\
$f_{0}(980)$ & -535.1 & $f_{0}(1500)$ & -20.1 & 0.8157 & 0.5784 \\
\hline \hline
\end{tabular}

explain the small masses of the light nonet without identifying the other states in the heavy nonet.

(2) Secondly, there is a strong mixing between $|000\rangle$ and $|011\rangle$ through the color-spin interaction. The mixing term, $\left\langle 000\left|V_{\mathrm{CS}}\right| 011\right\rangle$, is found to be very large, which, under the diagonalization of the hyperfine mass matrix, separates physical hyperfine masses by about $500 \mathrm{MeV}$ or more between the two nonets. (See Table II.) This is qualitatively consistent with the huge mass gaps, more than $500 \mathrm{MeV}$ or so, existing between the two nonets in PDG.

(3) A more direct outcome that can be tested in experiments is a peculiar characteristic in their decay modes entirely from the tetraquark mixing framework. When the two tetraquarks, $|000\rangle$ and $|011\rangle$, decay into two pseudoscalar mesons through the fall-apart mechanism, our mixing framework predicts that the relative coupling strengths are enhanced for the light nonet while they are suppressed for the heavy nonet. In fact, this prediction is tested very well in the isovector channel, $a_{0}(980)$ and $a_{0}(1450)$ [37], in comparison with their experimental partial widths. 


\section{FURTHER TETRAQUARK SIGNATURES FOR THE TWO NONETS}

Maybe one possible objection to the tetraquark mixing framework is the assumption that the heavy nonet is the tetraquarks with flavor nonet similar to the light nonet. To solidify this assumption, it is necessary to collect all the tetraquark signatures. The quantum numbers of the two nonets, $J^{P C}=0^{++}, I=0,1 / 2,1$, can be one of the signatures because they coincide with those of the tetraquarks $|000\rangle$ and $|011\rangle$. But these quantum numbers can be generated also from other pictures like a two-quark picture with orbital angular momentum $\ell=1$ so they are not the signatures exclusively for the tetraquarks. More concrete signatures are the items (2), (3) in Sec. II which say that the mass splittings and the fall-apart modes of the nonet members are consistent with the tetraquark mixing framework. In this section, we study additional tetraquark signatures for the two nonets. The first part presents that the two nonets really form the flavor nonets with the mass ordering consistent with the tetraquarks. The second part is to show that our tetraquark mixing framework can explain partially the marginal mass ordering seen in the heavy nonet. In addition, we discuss the mixing parameters from the tetraquark mixing framework as an additional signature for the tetraquark.

\section{A. Two nonets as tetraquarks with the flavor nonet}

Let us begin by a discussion that the two nonets actually form the flavor nonets of $\mathrm{SU}(3)_{f}$. Apart from the quantum numbers, $J^{P C}=0^{++}, I=0,1 / 2,1$, a more direct sign to support the flavor nonet can be seen from the Gell-Mann-Okubo (GMO) mass relation. Using the experimental masses provided in Table I, we find that the light octet satisfies the GMO relation, $M^{2}\left[a_{0}(980)\right]+$ $3 M^{2}\left[f_{0}(500)\right] \approx 4 M^{2}\left[K_{0}^{*}(800)\right]$, within $14 \%{ }^{4}$. In addition, $f_{0}(980)$ can be taken as another isoscalar belonging to the nonet simply because it is heavier than the octet counterpart, $f_{0}(500)$. The heavy octet also satisfies the GMO relation, $M^{2}\left[a_{0}(1450)\right]+3 M^{2}\left[f_{0}(1370)\right] \approx 4 M^{2}\left[K_{0}^{*}(1430)\right]$, within $6 \%$. $^{5}$ The other isoscalar $f_{0}(1500)$ is taken as a nonet member because it is heavier than $f_{0}(1370)$. Therefore, the GMO relation provides supporting evidence in taking the two nonets in PDG as the flavor nonets of $\mathrm{SU}(3)_{f}$. Our selection for $f_{0}(1370)$ and $f_{0}(1500)$ may require further clarification because an alternative suggestion can be found in Ref. [55] where $f_{0}(1500)$ is a light glueball mixed with tetraquarks $f_{0}(1370)$ and $f_{0}(1710)$. But the lattice calculation [64] suggests that the scalar glueball is in good agreement with the observed properties of $f_{0}(1710)$.

\footnotetext{
${ }^{4}$ For the $f_{0}(500)$ mass, we take the central value from the mass range, 400-550 MeV, given in Table I.

${ }^{5}$ Again, for the $f_{0}(1370)$ mass, we take the central value from its experimental mass range, 1200-1500 MeV.
}

The GMO relation only shows that the two nonets form the flavor nonets but it does not determine whether the flavor nonets belong to a two-quark system or a four-quark system. More important characteristics in identifying the two nonets as the tetraquark nonets are the mass ordering among the nonet members. Here we reexamine this mass ordering in detail by considering the quark-mass contribution to the resonance masses.

Using the flavor wave functions given in Fig. 1 while assuming that $m_{u}=m_{d} \neq m_{s}$, it is straightforward to evaluate the quark mass contribution, $\sum m_{q}$, to the mass of each isospin member as

$$
\begin{gathered}
\sum m_{q}^{(I=1)}=2 m_{s}+2 m_{u}, \\
\sum m_{q}^{(I=1 / 2)}=m_{s}+3 m_{u}, \\
\sum m_{q}^{(I=0)}\left(\mathbf{8}_{f}\right)=\frac{2}{3}\left(m_{s}+5 m_{u}\right) .
\end{gathered}
$$

In the last equation, $\sum m_{q}^{(I=0)}\left(\mathbf{8}_{f}\right)$ denotes the quark mass contribution to the isoscalar resonance belonging to the flavor octet. Now, by imposing $m_{s}>m_{u}$, one can establish the mass ordering among the quark mass contributions,

$$
\sum m_{q}^{(I=1)}>\sum m_{q}^{(I=1 / 2)}>\sum m_{q}^{(I=0)}\left(\mathbf{8}_{f}\right) .
$$

This ordering should be maintained for the octet masses so that our tetraquarks have the mass ordering

$$
M_{I=1}>M_{I=1 / 2}>M_{I=0}\left(\mathbf{8}_{f}\right),
$$

among the octet members with definite isospins. This mass ordering, commonly known as "inverted spectrum," is a unique characteristic of the tetraquarks clearly distinguished from a two-quark picture $(q \bar{q})$ which generates the opposite ordering, $M_{I=1}<M_{I=1 / 2}<M_{I=0}\left(\mathbf{8}_{f}\right)$, like the mass ordering seen in the pseudoscalar resonances, $m_{\pi}<m_{K}<m_{\eta}$.

The other isoscalar member belonging to the flavor singlet has not been listed in the ordering [Eq. (12)] because of the following reasons. Its mass $M_{I=0}\left(\mathbf{1}_{f}\right)$ lies between $M_{I=1}$ and $M_{I=1 / 2}$ if the ordering is governed by the quark mass term. But the isoscalar masses can be further modified by the flavor mixing. The flavor mixing, as it separates strange quarks from up and down quarks in the wave functions, raises the flavor singlet mass, $M_{I=0}\left(\mathbf{1}_{f}\right)$, while it pushes down the flavor octet mass, $M_{I=0}\left(\mathbf{8}_{f}\right)$. However, even under the flavor mixing, the ordering above [Eq. (12)] is still maintained whereas the position of $M_{I=0}\left(\mathbf{1}_{f}\right)$ in the ordering is slightly obscured.

For the light nonet, this mass ordering is clearly exhibited through $M\left[a_{0}(980)\right]>M\left[K_{0}^{*}(800)\right]>M\left[f_{0}(500)\right]$ as one can see from Table I. The other isoscalar $f_{0}(980)$ can be taken 
as a nonet member because it is heavier than $f_{0}(500)$ and $K_{0}^{*}(800)$. In fact, it is well known that the mass ordering provides a clear ground in identifying the light nonet as tetraquarks $[27,28]$. One may argue that the light nonet masses are rather small to be a four-quark state. But, as we showed in Table II, the hyperfine masses for the light nonet calculated from the tetraquark mixing framework are huge negative numbers in the range $-670 \sim-490 \mathrm{MeV}$, so that they can qualitatively explain the smallness of the light nonet masses.

This mass ordering [Eq. (12)] is also maintained in the heavy nonet. The isovector member in the heavy nonet, $a_{0}(1450)$, is slightly heavier than the isodoublet member, $K_{0}^{*}(1430)$, only by $50 \mathrm{MeV} . K_{0}^{*}(1430)$ is heavier than the isoscalar $f_{0}(1370)$ if its central value is taken from the experimentally known mass range $1200-1500 \mathrm{MeV}$. (See Table I.) So even though the mass ordering is marginal, one can assume the heavy nonet as tetraquarks. Indeed, our tetraquark mixing framework in Refs. [36-38], where the heavy nonet is assumed to be tetraquarks, provides nice phenomenological agreements in terms of mass splitting and decay strengths as summarized in Sec. II. However, because of the marginal mass ordering, the heavy nonet may require further supports in treating its members as tetraquarks.

\section{B. Hyperfine mass ordering}

A related issue to the mass ordering is the ordering among the hyperfine masses which can support our identification for the heavy nonet. As one can see in Table II, our tetraquark mixing framework generates the hyperfine masses which are ordered as $\left\langle V_{\mathrm{CS}}\right\rangle_{I=1}>\left\langle V_{\mathrm{CS}}\right\rangle_{I=1 / 2}>\left\langle V_{\mathrm{CS}}\right\rangle_{I=0}\left(\mathbf{8}_{f}\right)$. This ordering holds for the two nonets. Note that this hyperfine mass ordering is the same as the mass ordering [Eq. (12)]. This means that the mass ordering among the octet members is generated not only by the quark masses but also by the hyperfine masses.

But the magnitude of the spitting among the isospin members is quite different depending on the light and heavy nonets. Their splittings in $\mathrm{MeV}$ units obtained from Table II are

\begin{tabular}{l|c|c}
\hline & Light nonet & Heavy nonet \\
\hline$\left\langle V_{\mathrm{CS}}\right\rangle_{I=1}-\left\langle V_{\mathrm{CS}}\right\rangle_{I=1 / 2}$ & 104.2 & 10.1 \\
$\left\langle V_{\mathrm{CS}}\right\rangle_{I=1 / 2}-\left\langle V_{\mathrm{CS}}\right\rangle_{I=0}\left(\mathbf{8}_{f}\right)$ & 74.8 & 2.3 \\
\hline
\end{tabular}

From this, we see that the hyperfine masses contribute to the mass ordering by $104 \mathrm{MeV}$ or $75 \mathrm{MeV}$ for the light nonet but they contribute only by $10 \mathrm{MeV}$ or $2 \mathrm{MeV}$ for the heavy nonet. That is, the hyperfine mass splitting among the heavy nonet members is substantially narrower than the splitting among the light nonet members so subsequently the mass ordering in the heavy nonet must be narrower by $94 \mathrm{MeV}$ or $73 \mathrm{MeV}$. This narrowing down in the hyperfine mass splitting can provide a partial explanation on the marginal mass ordering seen in the heavy nonet. Since this is a direct consequence of the mixing formulas, Eqs. (6) and (7), this result could be another support for our tetraquark mixing framework that has not been pointed out in our previous works [36-38].

But we admit that such a narrowing down in hyperfine masses is not enough to explain the marginal mass ordering fully. For a full description, it may be necessary to include a two-quark component in the heavy nonet and its mixing with the tetraquarks in order to compensate for the additional gap in the opposite mass ordering generated from the twoquark component. But this program may require anomalous interactions with the flavor determinant as the two-quark and tetraquark components do not mix under the color-spin or color-electric interactions. Anyway, based on the successful aspects seen in the tetraquark mixing framework, we expect that the two-quark contribution is small. Nevertheless, this constitutes interesting future work to do.

\section{Mixing parameters}

Another signature to support the flavor nonet from the tetraquark mixing framework can be seen through the mixing parameters, $\alpha, \beta$, in Eqs. (6) and (7). The mixing parameters, $\alpha, \beta$, are fixed by the diagonalization of the hyperfine mass matrix in each isospin channel. Their values, as they are determined in each isospin channel independently, must depend on isospins in principle. But as one can see in Table II, the sensitivity to isospins is very small and in fact they are approximately close to the common values $\alpha \approx \sqrt{2 / 3}=0.8165, \beta \approx \sqrt{1 / 3}=0.5774$. The common mixing parameters are interesting given the fact that the associated hyperfine masses have a noticeable dependence on isospins as one can see in Table II.

These common mixing parameters imply that the members in each nonet, given by either Eq. (6) or Eq. (7), are related by a $\mathrm{SU}(3)_{f}$ rotation. For example, an isovector member can be obtained by a $\mathrm{SU}(3)_{f}$ rotation from an isodoublet member because both members are represented approximately by the same mixing parameters, $\alpha, \beta$. In other words, the left-hand sides of Eqs. (6) and (7) form nonets governed approximately by $\mathrm{SU}(3)_{f}$ just like $|000\rangle$, |011〉 do. This is quite consistent with the phenomenological fact that the two nonets in PDG form the flavor nonets through the GMO relation or the mass ordering. Therefore, our result from the common mixing parameters provides another signature to support the tetraquark mixing framework.

\section{OTHER PICTURES FOR THE TWO NONETS}

One may try different pictures other than tetraquarks in explaining the two nonets. A two-quark picture $(q \bar{q})$ with $\ell=1$ is one simple scenario as this can generate the same quantum numbers of the two nonets. An alternative picture that is often discussed in the literature is hadronic 
molecules of meson-meson bound states. In this section, we discuss the other pictures, how their predictions are different from the tetraquark picture, and what the possible problems are. So the purpose of the discussion here is to give more orientation toward a tetraquark description for the two nonets.

\section{A. Two-quark picture with $\ell=1$}

It is true that a two-quark $(q \bar{q})$ picture with $\ell=1$ can generate a nonet with the quantum numbers $J^{P}=0^{+}$. But we want to address that this picture does not fit well to the two nonets in PDG. First of all, the number of possible configurations with the $q \bar{q}(\ell=1)$ picture is restricted to one so that this picture cannot make the two nonets in the $J^{P}=0^{+}$channel. More precisely, when the spin of $q \bar{q}$, which we denote by $S$, is combined with the orbital angular momentum $\ell=1$, this picture generates various nonets belonging to the total angular momentum, $J=0,1,2$, with the following configurations:

$$
\begin{gathered}
J=0:(S=1, \ell=1), \\
J=1:(S=0, \ell=1),(S=1, \ell=1), \\
J=2:(S=1, \ell=1) .
\end{gathered}
$$

In this two-quark picture, the $J=0$ nonet must correspond to the two nonets in PDG but, as shown in Eq. (13), we have only one configuration ( $S=1, \ell=1$ ), which is obviously not enough to explain the two nonets listed in PDG. So the two nonets in PDG cannot be explained by the two-quark picture.

Alternatively, one may adopt different pictures for the light and heavy nonets. Specifically, one can assume that the light nonet is pure tetraquarks while the heavy nonet is described by a two-quark system with $\ell=1$. This assumption is based on an observation that the light nonet clearly exhibits a well-separated mass ordering consistent with the tetraquarks while the heavy nonet has the marginal ordering so that its tetraquark structure may be slightly obscured. So, one may try a $q \bar{q}(\ell=1)$ picture only for the heavy nonet.

If the heavy nonet is viewed as a $q \bar{q}(\ell=1)$, this nonet must have the configuration $(S=1, \ell=1)$ in making the states with the total angular momentum, $J=0$. This implies that the resonances in the heavy nonet can be regarded as the states orbitally excited from the spin-1 vector mesons, $\rho, K^{*}$, $\omega, \phi$. Thus, the heavy nonet masses relative to the lowestlying vector nonet must be generated by the spin-orbit interaction. Problems with this two-quark picture can be seen from the mass gaps between the $I=1 / 2, I=1$ members before and after turning on the spin-orbit interactions. As shown in Fig. 2, its gap in the lowest-lying vector nonet is $M\left[K^{*}(892)\right]-M[\rho(770)] \approx 116 \mathrm{MeV}$, with the isodoublet member being heavier. Obviously, this
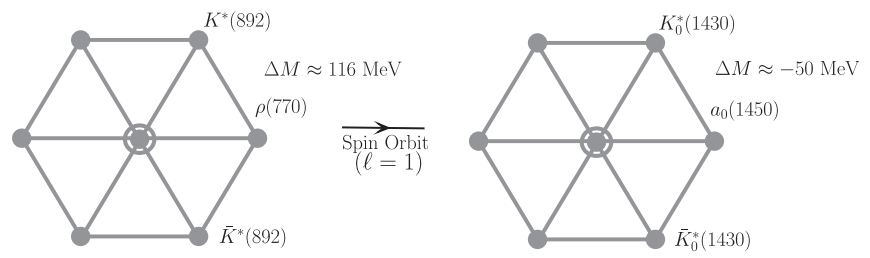

FIG. 2. A schematic picture to generate the heavy nonet when its members are viewed as orbital excitations of the $q \bar{q}$ vector nonet.

mass ordering is driven by the strange quark being heavier than the up or down quark. On the other hand, the corresponding mass gap in the heavy nonet, $M\left[K_{0}^{*}(1430)\right]-M\left[a_{0}(1450)\right] \approx-50 \mathrm{MeV}$, exhibits that the isovector member is heavier. To explain this, the spinorbit interaction must contribute very differently to the $I=1, I=1 / 2$ members so that it even flips the mass ordering established by the heavy strange quark mass. We believe that this picture is not realistic for the heavy nonet.

Another approach is to introduce a mixing framework between the $q \bar{q}(\ell=1)$ and the tetraquark picture. The marginal mass ordering seen in the heavy nonet may have room for a two-quark component to be included. One way to accomplish this is to introduce a mixing between the $q \bar{q}$ $(\ell=1)$ states with the tetraquark $(q q \bar{q} \bar{q})$ states in generating the two nonets in PDG. This is possible in principle because one can construct a $\mathrm{SU}(3)_{f}$ invariant among the two nonets represented by effective hadronic fields for the $q \bar{q}$ and for the $q q \bar{q} \bar{q}$ [57]. But, as pointed out by Ref. [55], the required mixing magnitude seems unnaturally large given the fact that the very different configurations are involved. In reality, the $q \bar{q}(\ell=1)$ states do not mix with the $q q \bar{q} \bar{q}$ states through the color-spin and color-electric interactions which are, however, believed to be the major interactions among quarks inside hadrons.

\section{B. Meson-meson picture}

Another picture to describe the two nonets in PDG is the meson-meson bound states where two colorless mesons are bound by the color residual forces just like a deuteron of a proton-neutron system. These molecular states are expected to have less binding than the tetraquarks whose binding is provided by direct color forces between the two colorful objects, diquark and antidiquark. Because of this, the meson-meson states, if they exist, must form shallow bound states. This means that the meson-meson bound states can be characterized by their mass close to the sum of their constituent meson masses [45,50]. A typical example along this line is given by Ref. [48] where it is argued that the $X(3872)$ mass, which is close to the $D^{0} \bar{D}^{* 0}$ threshold, may be an indication of its molecular nature. One may adopt a similar picture for the light and heavy nonet members. In particular, $f_{0}(980)$ and $a_{0}(980)$ in the light nonet may be the shallow bound states of $K \bar{K}$ [41-44] 
because their masses are in the proximity of $2 M_{K}$. Also for the heavy nonet, one can build the shallow bound states from two vector mesons whose total masses are not far from masses of the heavy nonet members. But this molecular picture may not be applied to all the members in both nonets. For example, $f_{0}(500)$ in the light nonet is hard to consider as a shallow bound state of $\pi \pi$ due to the large mass difference.

In practice, the meson-meson picture tends to involve model dependence as its description relies on some phenomenological pictures such as the one pion exchange potential. Binding mechanisms as well as the corresponding energies may not be determined unanimously. So it is difficult to consider this picture conclusive. Another problem with the meson-meson picture is the absence of the additional multiplets expected in the hadron spectrum. The bound states from the meson octet-meson octet form various multiplets through the group multiplication, $\mathbf{8} \otimes \mathbf{8}=\mathbf{2 7} \oplus \mathbf{1 0} \oplus \overline{\mathbf{1 0}} \oplus \mathbf{8} \oplus \mathbf{8} \oplus \mathbf{1}$. The color residual forces that bind these mesons are of the same type because the constituent mesons are always in the color-singlet state of $\sim \bar{q}^{a} q_{a}$. In other words, the color residual forces do not discriminate much in forming all the possible multiplets. So, if this meson-meson picture works, we expect the additional resonances with the higher isospins $I=2,3 / 2$ belonging to 27,10 or $\overline{\mathbf{1 0}}$ in addition to the two nonets with $I=0,1 / 2,1$. But this expectation is not supported by the current PDG. Even the states with $I=0,1 / 2,1$ need to be more numerous than what we currently have in PDG as the higher multiplets can generate those isospin states also. (See Table I in Ref. [38] for the $0^{+}$resonances in PDG.) One possible resolution to this discrepancy can be found from Refs. [65,66] where the attraction in those exotic channels, calculated through Weinberg-Tomozawa term in the flavor $\mathrm{SU}(3)_{f}$ symmetric limit, is not strong enough to generate bound states. So the meson-meson picture may have some room to accommodate the two nonets still.

Our tetraquark mixing model has one common feature with this meson-meson picture in that the tetraquark wave functions also have two-meson channels which can fall apart into either two pseudoscalar or two vector mesons. The difference is that the two-meson channels are only the part of the wave functions in the tetraquark picture while they are the full component in the meson-meson picture. In addition, the two-meson channels are close to on-shell in the meson-meson picture while they can be extended to offshell in the tetraquark picture. More importantly, the tetraquark mixing model predicts quite different coupling strengths for the two-pseudoscalar modes depending on the light or heavy nonet $[37,38]$. This could be a unique consequence of the tetraquark mixing framework generated by Eqs. (6) and (7), probably not present in the mesonmeson picture as there is no similar mixing mechanism. As we will examine below in Sec. V, one can establish similar phenomena in the two vector couplings with quite different characteristics. Eventually, these types of studies can be used to determine a realistic picture for the two nonets, meson-meson picture or tetraquark mixing picture.

\section{FALL-APART MODES INTO TWO VECTOR MESONS}

Our tetraquark mixing framework can be succinctly represented by Eqs. (6) and (7). Due to the relative sign difference in the two equations, the two tetraquark types, $|000\rangle$ and $|011\rangle$, partially cancel in making the heavy nonet while they add up in making the light nonet. This in fact leads to an interesting phenomenon in the fall-apart strengths into two pseudoscalar mesons [37]. Specifically, the corresponding coupling strengths are suppressed for the heavy nonet while they are enhanced for the light nonet. In this section, we look for another signature for the tetraquark mixing framework from the fall-apart modes into the two vector mesons.

First, the appearance of two-meson states can be demonstrated easily by rewriting the tetraquark wave functions with respect to the quark and antiquark bases. To illustrate this, let us label the tetraquarks in the diquarkantidiquark picture as $q_{1} q_{2} \bar{q}_{3} \bar{q}_{4}$ and rewrite them by recombining quarks and antiquarks into the 13- and 24pair. ${ }^{6}$ This recombination in color space is schematically represented by

$$
\left(q_{1} q_{2} \bar{q}_{3} \bar{q}_{4}\right)_{\mathbf{1}_{c}} \sim\left[\mathbf{8}_{c 13} \otimes \mathbf{8}_{c 24}\right]_{\mathbf{1}_{c}}+\left[\mathbf{1}_{c 13} \otimes \mathbf{1}_{c 24}\right]_{\mathbf{1}_{c}} .
$$

Here the notation such as $\mathbf{8}_{c 13}$ denotes the 13-pair of the quark-antiquark in the color octet. From this equation, we notice that the tetraquarks have two components that differ by the color configurations in the quark-antiquark bases. The first component is composed of two pairs of a quarkantiquark, both belonging to $\mathbf{8}_{c}$ in forming a color-singlet totally. The second component contains the two pairs belonging to $\mathbf{1}_{c}$.

It is this second component that corresponds to twomeson modes which can fall apart into two mesons if the decays are kinematically allowed. Specifically the inner product of a two-meson state with Eq. (16) picks out the same state from the second component with the corresponding relative coupling determined by the color, spin, and flavor recombination factors. Thus, to find the relative strengths of the fall-apart modes, one needs to calculate the numerical factors coming from color, spin and flavor recombination separately in the second term of Eq. (16). The purpose of this section is to provide technical details in calculating possible modes and their relative strengths in two vector channels.

\footnotetext{
${ }^{6}$ The other recombination into the 14- and 23-pair gives the same fall-apart modes.
} 


\section{A. Color and spin factors in the recombination}

Let us start with the color factors in this recombination of the tetraquark wave functions. To form two mesons in the final states, the 13- and 24-pair in Eqs. (2) and (4) must be in a color-singlet state separately. By isolating the colorsinglet pieces, and substituting them into Eqs. (2) and (4), one arrives at the following replacements:

$$
\begin{aligned}
\frac{1}{\sqrt{12}} \varepsilon_{a b d} \varepsilon_{a e f}\left(q_{1}^{b} q_{2}^{d}\right)\left(\bar{q}_{3}^{e} \bar{q}_{4}^{f}\right) & \rightarrow \frac{1}{\sqrt{3}} \mathbf{1}_{c 13} \mathbf{1}_{c 24}, \\
\frac{1}{\sqrt{96}}\left(q_{1}^{a} q_{2}^{b}+q_{1}^{b} q_{2}^{a}\right)\left(\bar{q}_{3}^{a} \bar{q}_{4}^{b}+\bar{q}_{3}^{b} \bar{q}_{4}^{a}\right) & \rightarrow \sqrt{\frac{2}{3}} \mathbf{1}_{c 13} \mathbf{1}_{c 24} .
\end{aligned}
$$

This shows that the recombination color factor is $\sqrt{1 / 3}$ from the first tetraquark type, $|000\rangle$, and $\sqrt{2 / 3}$ from the second tetraquark type, $|011\rangle$. An additional sign expected from anticommuting fermion fields does not affect our results because it changes only the overall sign for both Eqs. (17) and (18). Our results in the fall-apart modes depend only on the relative signs.

One thing to point out is that the color recombination factors are approximately close to the tetraquark mixing parameters, $\alpha \approx \sqrt{2 / 3}$ and $\beta \approx \sqrt{1 / 3}$. If these numbers are inserted into Eq. (6), the fall-apart modes almost cancel, eventually yielding zero coupling strengths to the heavy nonet. Thus, as far as the color factors are concerned, the fall-apart modes from the heavy nonet vanish approximately. However, this expectation does not occur when the additional factors coming from the spin recombination below are included.

Next, we calculate the spin factors in this recombination. The first tetraquark has the spin configuration, $\left|J J_{12} J_{34}\right\rangle=$ $|000\rangle$. The diquark, since its spin $J_{12}$ and the spin projection $M_{12}$ are zero, is in the state, $\left|J_{12} M_{12}\right\rangle=|0,0\rangle_{12}$. And the antidiquark is in $\left|J_{34} M_{34}\right\rangle=|0,0\rangle_{34}$. This means that the first tetraquark can be written as

$$
|000\rangle=|0,0\rangle_{12}|0,0\rangle_{34},
$$

in terms of the diquark (antidiquark) spin and its projection. Since $|0,0\rangle_{12}=\frac{1}{\sqrt{2}}\left[\uparrow_{1} \downarrow_{2}-\downarrow_{1} \uparrow_{2}\right],|0,0\rangle_{34}=\frac{1}{\sqrt{2}}\left[\uparrow_{3} \downarrow_{4}-\downarrow_{3} \uparrow_{4}\right]$, one can rewrite $|000\rangle$ in terms of the individual quark spinors as

$$
|000\rangle=\frac{1}{2}\left[\uparrow_{1} \downarrow_{2}-\downarrow_{1} \uparrow_{2}\right]\left[\uparrow_{3} \downarrow_{4}-\downarrow_{3} \uparrow_{4}\right] .
$$

The 13-pairs in the right-hand side can be expressed by the definite spin states, $\left|J_{13} M_{13}\right\rangle$, as

$$
\begin{gathered}
\uparrow_{1} \uparrow_{3}=|1,1\rangle_{13}, \\
\uparrow_{1} \downarrow_{3}=\frac{1}{\sqrt{2}}\left[|1,0\rangle_{13}+|0,0\rangle_{13}\right],
\end{gathered}
$$

$$
\begin{gathered}
\downarrow_{1} \uparrow_{3}=\frac{1}{\sqrt{2}}\left[|1,0\rangle_{13}-|0,0\rangle_{13}\right], \\
\downarrow_{1} \downarrow_{3}=|1,-1\rangle_{13} .
\end{gathered}
$$

The 24-pairs in Eq. (20) can be written similarly by its spin states, $|1,1\rangle_{24},|1,0\rangle_{24},|1,-1\rangle_{24}$, and $|0,0\rangle_{24}$.

Putting all these into Eq. (20), we obtain the final expression for $|000\rangle$ with respect to the spin states of the 13- and 24-pair:

$$
\begin{aligned}
|000\rangle= & \frac{1}{2}\left[|1,1\rangle_{13}|1,-1\rangle_{24}-|1,0\rangle_{13}|1,0\rangle_{24}\right. \\
& \left.+|0,0\rangle_{13}|0,0\rangle_{24}+|1,-1\rangle_{13}|1,1\rangle_{24}\right] .
\end{aligned}
$$

The subscripts 13 and 24 denote the quark-antiquark pairs so each term in the right-hand side corresponds to twomeson channels with the designated spin states. The component, $|0,0\rangle_{13}|0,0\rangle_{24}$, corresponds to two pseudoscalar mesons and the other three components, $|1,1\rangle_{13}|1,-1\rangle_{24}$, $|1,0\rangle_{13}|1,0\rangle_{24}$, and $|1,-1\rangle_{13}|1,1\rangle_{24}$, correspond to two vector-mesons distinguished by the spin projections. These modes can be measured experimentally if the invariant masses of two mesons are less than the tetraquark masses. By defining the two pseudoscalar and two vector parts as

$$
\begin{gathered}
P P=|0,0\rangle_{13}|0,0\rangle_{24} \\
V V=\frac{1}{\sqrt{3}}\left[|1,1\rangle_{13}|1,-1\rangle_{24}-|1,0\rangle_{13}|1,0\rangle_{24}\right. \\
\left.+|1,-1\rangle_{13}|1,1\rangle_{24}\right]
\end{gathered}
$$

one can rewrite Eq. (25) neatly as

$$
|000\rangle=\frac{1}{2} P P+\frac{\sqrt{3}}{2} V V .
$$

The second tetraquarks have the spin configuration, $|011\rangle$, containing a spin-1 diquark and spin-1 antidiquark with three possible projections. The four-quark state, as its spin and projection are zero, can be expressed by the spin states of diquark and antidiquark as

$$
\begin{aligned}
|011\rangle= & \frac{1}{\sqrt{3}}\left\{|1,1\rangle_{12}|1,-1\rangle_{34}-|1,0\rangle_{12}|1,0\rangle_{34}\right. \\
& \left.\left.+|1,-1\rangle_{12}|1,1\rangle_{34}\right]\right\},
\end{aligned}
$$

including the relevant Clebsch-Gordan coefficients. The rearrangement into the 13- and 24-pair can be done similarly as before by using Eqs. (21)-(24). Skipping all the details, we simply write down the final expression, 


$$
\begin{aligned}
|011\rangle= & \frac{1}{2 \sqrt{3}}\left\{-|1,1\rangle_{13}|1,-1\rangle_{24}+|1,0\rangle_{13}|1,0\rangle_{24}\right. \\
& \left.+3|0,0\rangle_{13}|0,0\rangle_{24}-|1,-1\rangle_{13}|1,1\rangle_{24}\right\} \\
= & \frac{\sqrt{3}}{2} P P-\frac{1}{2} V V .
\end{aligned}
$$

Similarly as Eq. (28), this second tetraquark can couple to two pseudoscalar mesons and two vector mesons also.

Comparing Eqs. (28) and (30), one can see that the twopseudoscalar mode, $P P$, has the same sign in both equations but with different numerical factors. Substituting Eqs. (28) and (30) into Eq. (6), we see in the heavy nonet that the cancellation still occurs for the two-pseudoscalar modes but partially. For the light nonet, by inserting Eqs. (28) and (30) into Eq. (7), we see that the two-pseudoscalar modes add up to each other. This difference eventually makes the associated couplings suppressed for the heavy nonet and enhanced for the light nonet $[37,38]$.

On the other hand, the two-vector mode, $V V$, has the opposite sign in Eqs. (28) and (30). In this case, we find analogous results but with the opposite trend. Namely, the two-vector modes add up in the heavy nonet, enhancing their associated couplings, while they cancel each other in the light nonet, suppressing their couplings. So these characteristics in the two-vector modes are clearly different from those of the two-pseudoscalar modes.

By combining the color factors in Eqs. (17) and (18) with the spin factors in Eqs. (28) and (30), we obtain the corresponding factors for the two-vector modes,

$$
\begin{gathered}
|000\rangle \rightarrow \frac{1}{\sqrt{3}} \times \frac{\sqrt{3}}{2}=\frac{1}{2}, \\
|011\rangle \rightarrow \sqrt{\frac{2}{3}} \times\left(-\frac{1}{2}\right)=-\frac{1}{\sqrt{6}},
\end{gathered}
$$

from the two tetraquark types. Inserting these into Eqs. (6) and (7), we finally obtain the necessary factors coming from the color and spin recombination for the heavy and light nonet, respectively, as

$$
\begin{aligned}
\mid \text { Heavy nonet }\rangle & \rightarrow-\frac{\alpha}{2}-\frac{\beta}{\sqrt{6}}, \\
\mid \text { Light nonet }\rangle & \rightarrow \frac{\beta}{2}-\frac{\alpha}{\sqrt{6}} .
\end{aligned}
$$

As one can see from the relative signs, the two terms add up in Eq. (33), while the corresponding terms cancel partially in Eq. (34). This form of the color and spin factors is common for all the members in each nonet even though $\alpha, \beta$ have a slight isospin dependence as shown in Table II. Therefore, as advertised, the two-vector modes are enhanced for the heavy nonet while they are suppressed for the light nonet.

\section{B. Flavor factors in the recombination}

To find concrete two-meson channels, we now move to the flavor recombination in terms of the 13- and 24-pair. Our tetraquarks have the flavor structures given in Fig. 1 and they are the same for $|000\rangle,|011\rangle$ by construction. In order to find the fall-apart modes into the two vector mesons, we start by writing the $q \bar{q}$ representation for the vector mesons,

$$
\begin{gathered}
u \bar{s}=K^{*+}, \quad d \bar{s}=K^{* 0}, \quad s \bar{u}=\bar{K}^{*-}, \quad s \bar{d}=\bar{K}^{* 0}, \\
s \bar{s}=\phi, \quad u \bar{u}=\frac{1}{\sqrt{2}}\left(\omega+\rho^{0}\right), \quad d \bar{d}=\frac{1}{\sqrt{2}}\left(\omega-\rho^{0}\right), \\
u \bar{d}=\rho^{+}, \quad d \bar{u}=\rho^{-} .
\end{gathered}
$$

Here the flavor mixing is assumed to occur ideally according to the Okubo-Zweig-Iizuka (OZI) rule so that the strange quark is completely decoupled from the up and down quarks among the two isoscalar members, $\phi$ and $\omega$.

Now for the isovector members in our tetraquarks, we have $a_{0}^{+}(980)$ in the light nonet and $a_{0}^{+}(1450)$ in the heavy nonet. ${ }^{7}$ Both have the common flavor structure $[s u][\bar{d} \bar{s}]$. In the recombination into the 13- and 24-pair, it becomes $(s \bar{d})(u \bar{s})-(s \bar{s})(u \bar{d})$. Using the identifications in Eq. (35), we find the following two-vector modes common for $a_{0}^{+}(980)$ and $a_{0}^{+}(1450)$ :

$$
\bar{K}^{* 0} K^{*+}-\phi \rho^{+} .
$$

For the isodoublet members, we have $K_{0}^{*+}(800)$ in the light nonet and $K_{0}^{*+}(1430)$ in the heavy nonet. Their flavor structure, which is common for both, is $[u d][\bar{d} \bar{s}]$. If it is rearranged into the quark-antiquark pairs, we find $(u \bar{d})(d \bar{s})-$ $(u \bar{s})(d \bar{d})$. Again the representation in Eq. (35) leads to the two-vector modes common for $K_{0}^{*+}(800)$ and $K_{0}^{*+}(1430)$ :

$$
\rho^{+} K^{* 0}-\frac{1}{\sqrt{2}} K^{*+}\left[\omega-\rho^{0}\right] .
$$

For the isoscalar members, we have two sets of resonances. One set is $f_{0}(500)$ and $f_{0}(1370)$, which are close to the flavor octet members belonging separately to the two nonets, and the other set is $f_{0}(980)$ and $f_{0}(1500)$, which are close to the flavor singlet members. The two resonances in each set have the same flavor structure. The reason why we call these resonances as "close to the octet or singlet" is because they are subject to the flavor mixing according to the OZI rule generalized to the four-quark system. ${ }^{8}$ In other words, they are not definite members of SU(3) $)_{f}$. The flavor

\footnotetext{
${ }^{7}$ We choose the positive-charged state here but our result should be the same for the other isospin members as they are simply related by isospin rotations.

${ }^{8}$ This type of generalization of the OZI rule has been applied also to pentaquarks [67].
} 
mixing occurs among the light nonet members, $f_{0}(500)$ and $f_{0}(980)$, and similarly among the heavy nonet members, $f_{0}(1370)$ and $f_{0}(1500)$.

In this generalization of the flavor mixing, the isoscalar resonances can be represented by linear combinations of the ideal mixing states, $|L\rangle|H\rangle$, defined by

$$
\begin{gathered}
|L\rangle=[u d][\bar{u} \bar{d}], \\
|H\rangle=\frac{1}{\sqrt{2}}\{[d s][\bar{d} \bar{s}]+[s u][\bar{s} \bar{u}]\} .
\end{gathered}
$$

Namely, the flavor structure of the first set of resonances, $f_{0}(500)$ and $f_{0}(1370)$, is given by the combination

$$
a|L\rangle+b|H\rangle,
$$

while the second set of resonances, $f_{0}(980)$ and $f_{0}(1500)$, have the flavor structure written by

$$
-b|L\rangle+a|H\rangle .
$$

The flavor mixing parameters, $a, b$, can be fixed depending on how we implement the flavor mixing [38]. When $a=\sqrt{2 / 3}, b=-\sqrt{1 / 3}$, we get the $\mathrm{SU}(3)_{f}$ symmetric case where there is no flavor mixing. When $a=1, b=0$, we get the ideal mixing case, i.e., $|L\rangle,|H\rangle$. In this case, the four-quark states containing strange quarks are completely separated from those states composed only by up and down quarks. The other case is the realistic case with fitting (RCF) [38] where we fit the flavor mixing parameters by tuning them to reproduce the mass splitting between $f_{0}(980)$ and $f_{0}(1500)$ equivalent to their hyperfine mass splitting. The flavor mixing parameters in RCF have been determined to be

$$
a=0.8908, \quad b=-0.4543 .
$$

Among the three cases, the physical isoscalar states in each nonet must be the ones that diagonalize the full Hamiltonian. Within our framework using the color-spin interaction, $V_{\mathrm{CS}}$, the two isoscalars in each nonet can be mixed and further diagonalization is necessary in order to generate the physical states. In fact, one can demonstrate that the ideal mixing states, $|L\rangle,|H\rangle$, are the eigenstates that diagonalize the color-spin interaction as $\left\langle L\left|V_{\mathrm{CS}}\right| H\right\rangle=0$. However, neither the $\mathrm{SU}(3)_{f}$ symmetric case nor the ideal mixing case represent the real situation for the isoscalars in the two nonets. In reality, there could be additional interactions like the anomalous term which is normally responsible for the mass splitting between $\eta$ and $\eta^{\prime}$. Such an interaction may exist also in our tetraquark system. But, according to our analysis for the decays into two pseudoscalar mesons [38], some modes in the ideal mixing case or in the $\mathrm{SU}(3)_{f}$ symmetric case are found to be inconsistent with the experimental modes. Moreover, the anomalous interaction introduces further ambiguity in the model as additional parameters entailed in this interaction may not be estimated reliably. Instead, RCF includes such an interaction indirectly as the flavor mixing parameters here are fitted to the physical mass difference of two isoscalar resonances. The RCF result seems to give a more consistent description of the two-pseudoscalar fall-apart modes in comparison with the PDG data. So in our fall-apart modes into two vector mesons, we will be considering this realistic case only.

Now, the fall-apart modes for the isoscalar resonances can be constructed from those for $|L\rangle,|H\rangle$ through Eqs. (40) and (41) with the mixing parameters given in Eq. (42). In fact, the fall-apart modes for $|L\rangle,|H\rangle$ are determined straightforwardly as

$$
\begin{gathered}
|L\rangle \Rightarrow \frac{1}{2}[\omega \omega-\boldsymbol{\rho} \cdot \boldsymbol{\rho}], \\
|H\rangle \Rightarrow \omega \phi-\frac{1}{\sqrt{2}} \bar{K}^{*} K^{*},
\end{gathered}
$$

with the shorthand notations for the isovector and isodoublet resonances defined by

$$
\begin{aligned}
\boldsymbol{\rho} \cdot \boldsymbol{\rho} & =\rho^{0} \rho^{0}+\rho^{+} \rho^{-}+\rho^{-} \rho^{+}, \\
\bar{K}^{*} K^{*} & =K^{*-} K^{*+}+\bar{K}^{* 0} K^{* 0} .
\end{aligned}
$$

Putting these modes into Eq. (40), we find the two-vector modes for $f_{0}(500)$ and $f_{0}(1370)$ as

$$
\frac{a}{2} \omega \omega+b \omega \phi-\frac{a}{2} \rho \cdot \rho-\frac{b}{\sqrt{2}} \bar{K}^{*} K^{*} .
$$

Note that, due to the isospin factors, the $\rho^{+} \rho^{-}$coupling is twice the $\rho^{0} \rho^{0}$ coupling and the $K^{*-} K^{*+}$ coupling is the same as the $\bar{K}^{* 0} K^{* 0}$ coupling.

The two-vector modes from the other members, $f_{0}(980)$ and $f_{0}(1500)$, are obtained from Eq. (45) simply by replacing $a \rightarrow-b, b \rightarrow a$,

$$
-\frac{b}{2} \omega \omega+a \omega \phi+\frac{b}{2} \rho \cdot \rho-\frac{a}{\sqrt{2}} \bar{K}^{*} K^{*},
$$

because their flavor structure is given by Eq. (41) that can be obtained from Eq. (40) with the same replacements.

\section{Total strengths of the fall-apart modes}

Relative strengths of the fall-apart modes into two vector mesons can be obtained by combining the spin and color recombination factor in Eq. (33) for the heavy nonet and in Eq. (34) for the light nonet, with the flavor recombination factor in Eq. (36) for the isovectors, in Eq. (37) for the isodoublets, and in Eqs. (45) and (46) for the isoscalar resonances. They are given by 


$$
\begin{aligned}
a_{0}^{+}(980):\left\{\bar{K}^{* 0} K^{*+}-\phi \rho^{+}\right\} \times\left(\frac{\beta}{2}-\frac{\alpha}{\sqrt{6}}\right), \\
a_{0}^{+}(1450):\left\{\bar{K}^{* 0} K^{*+}-\phi \rho^{+}\right\} \times\left(-\frac{\alpha}{2}-\frac{\beta}{\sqrt{6}}\right), \\
K_{0}^{*+}(800):\left\{\rho^{+} K^{* 0}-\frac{1}{\sqrt{2}} K^{*+}\left(\omega-\rho^{0}\right)\right\} \times\left(\frac{\beta}{2}-\frac{\alpha}{\sqrt{6}}\right), \\
K_{0}^{*+}(1430):\left\{\rho^{+} K^{* 0}-\frac{1}{\sqrt{2}} K^{*+}\left(\omega-\rho^{0}\right)\right\} \times\left(-\frac{\alpha}{2}-\frac{\beta}{\sqrt{6}}\right), \\
f_{0}(500):\left\{\frac{a}{2} \omega \omega+b \omega \phi-\frac{a}{2} \rho \cdot \rho-\frac{b}{\sqrt{2}} \bar{K}^{*} K^{*}\right\} \\
\times\left(\frac{\beta}{2}-\frac{\alpha}{\sqrt{6}}\right), \\
\times\left(-\frac{\alpha}{2}-\frac{\beta}{\sqrt{6}}\right) . \\
f_{0}(1500):\left\{-\frac{b}{2} \omega \omega+a \omega \phi+\frac{b}{2} \rho \cdot \rho-\frac{a}{\sqrt{2}} \bar{K}^{*} K^{*}\right\} \\
f_{0}(1370):\left\{\frac{a}{2} \omega \omega+b \omega \phi-\frac{a}{2} \rho \cdot \boldsymbol{\rho}-\frac{b}{\sqrt{2}} \bar{K}^{*} K^{*}\right\} \\
f_{0}(980):\left\{-\frac{\alpha}{2} \omega \omega+\frac{\beta}{\sqrt{6}}\right),
\end{aligned}
$$

For each isospin channel, the flavor recombination factor is the same for both nonets because the two nonets have the same flavor structure. So the fall-apart modes from the heavy and light nonets differ by color and spin recombination factors only.

The tetraquark mixing parameters, $\alpha, \beta$, depend on isospins and their numerical values can be found in Table II. The flavor mixing parameters, $a, b$, are given in Eq. (42). Using them, we present in Table III the numerical values for the fall-apart coupling strengths from all the resonances in the two nonets. It should be remembered that these are the relative strengths and the actual strengths are supposed to be multiplied by an unknown overall constant. Table III clearly shows that the relative strengths are enhanced for the heavy nonet while they are suppressed for the light nonet. The ratios of the two strengths, one from the light nonet and the other from the heavy nonet, become huge numbers around $\sim 15$ and
TABLE III. Here we present fall-apart modes into two-vector channels and their relative coupling strengths from the isovectors, $a_{0}^{+}(980)$ and $a_{0}^{+}(1450)$; the isodoublets, $K_{0}^{*+}(800)$ and $K_{0}^{*+}(1430)$; the isoscalars close to the flavor octet, $f_{0}(500)$ and $f_{0}(1370)$; and the isoscalars close to the flavor singlet, $f_{0}(980)$ and $f_{0}(1500)$. The ratios of the two strengths are also shown. To get the actual coupling strengths, it is necessary to multiply by a common unknown overall factor.

\begin{tabular}{l|c|c|c|c}
\hline \hline \multirow{4}{*}{$I=1$} & Mode & $a_{0}^{+}(980)$ & $a_{0}^{+}(1450)$ & Ratio \\
\cline { 2 - 5 } & $\bar{K}^{* 0} K^{*+}$ & -0.0449 & -0.6439 & 14.33 \\
\hline \multirow{5}{*}{$I=1 / 2$} & $\phi \rho^{+}$ & 0.0449 & 0.6439 & \\
\hline & Mode & $K_{0}^{*+}(800)$ & $K_{0}^{*+}(1430)$ & Ratio \\
\cline { 2 - 5 } & $\rho^{+} K^{* 0}$ & -0.0408 & -0.6442 & \\
& $\rho^{0} K^{*+}$ & -0.0289 & -0.4555 & 15.78 \\
& $\omega K^{*+}$ & 0.0289 & 0.4555 & \\
\hline \multirow{5}{*}{$I=0\left(\sim \mathbf{8}_{f}\right)$} & Mode & $f_{0}(500)$ & $f_{0}(1370)$ & Ratio \\
\cline { 2 - 5 } & $\rho^{0} \rho^{0}$ & 0.0185 & 0.2869 & \\
& $\bar{K}^{* 0} K^{* 0}$ & -0.0133 & -0.2069 & 15.54 \\
& $\phi \omega$ & 0.0188 & 0.2927 & \\
& $\omega \omega$ & -0.0185 & -0.2869 & \\
\hline \multirow{4}{*}{$I=0\left(\sim \mathbf{1}_{f}\right)$} & Mode & $f_{0}(980)$ & $f_{0}(1500)$ & Ratio \\
\cline { 2 - 5 } & $\rho^{0} \rho^{0}$ & 0.0100 & 0.1463 & \\
& $\bar{K}^{* 0} K^{* 0}$ & 0.0276 & 0.4057 & 14.70 \\
\hline \hline & $\phi \omega$ & -0.0390 & -0.5737 & \\
\hline & $\omega \omega$ & -0.0100 & -0.1463 & \\
\hline
\end{tabular}

they do not suffer from the unknown overall constant. One can also see that all the fall-apart modes in each isospin channel yield the same ratio. The ratio is 14.33 for $I=1$, 15.78 for $I=1 / 2,15.54$ for $I=0\left(\sim \mathbf{8}_{f}\right)$, and 14.70 for $I=0\left(\sim \mathbf{1}_{f}\right)$. This is a direct consequence of the fact that the two nonets have the same flavor structure. Namely, for each fall-apart mode, the heavy and light nonets have the same flavor recombination factor which is canceled away from the ratios. So the ratios are fixed purely by the spin and color recombination factors through Eq. (33) over Eq. (34).

This trend in the fall-apart strengths into two vector mesons, namely the enhancement for the heavy nonet and the suppression for the light nonet, is opposite to what we have found for the two-pseudoscalar modes whose fallapart strengths are suppressed for the heavy nonet but enhanced for the light nonet [37]. But both are the direct consequences of the tetraquark mixing framework, Eqs. (6) and (7). So these features are very promising in distinguishing the tetraquark mixing framework from other proposals for the two nonets in the literature. For the case of the twopseudoscalar modes, our results have been tested relatively well for $a_{0}(980)$ and $a_{0}(1450)$. That is, our calculated ratios of partial decay widths were found to reproduce successfully the experimental ratios [37]. This leads us to expect that the additional trend in the two-vector modes also exists as an actual phenomenon. 
However, unlike the two-pseudoscalar modes, the twovector modes are not directly accessible in most cases due to kinematical constraints. Most two-vector pairs from the fall-apart modes have invariant masses above the threshold set by the resonance masses belonging to the two nonets and, therefore, their partial widths cannot be measured experimentally. Instead, most coupling strengths presented in Table III can play the role of constraints when one constructs effective Lagrangians involving the participating mesons, which then can be used to investigate off-shell behaviors of the two nonets. They can be investigated, for example, in a coupled-channel analysis where the members in the two nonets appear in the intermediate states.

Nevertheless, the two decay modes from the heavy nonet, $f_{0}(1370) \rightarrow \rho \rho$ and $f_{0}(1500) \rightarrow \rho \rho$, should be interesting in comparison with experimental data. These two modes barely satisfy the kinematical constraints and provide indirect hints for the strength enhancement. According to PDG, $f_{0}(1370) \rightarrow \rho \rho$ is reported to be a dominant mode among various modes in the $f_{0}(1370) \rightarrow 4 \pi$ decays. Because of the invariant mass, $2 M_{\rho} \sim 1551 \mathrm{MeV}$, this decay occurs rarely only from high tails of the broad resonance $f_{0}(1370)$ and its partial width should be suppressed strongly by the limited phase space. So its dominance within the $4 \pi$ decay modes is difficult to understand unless there is a strong enhancement in the coupling with some understandable mechanisms. Indeed, the strong enhancement reported in Table III may provide one possible mechanism for this mode.

We have a similar enhancement for the $f_{0}(1500) \rightarrow \rho \rho$ mode in Table III. The partial decay width of this mode is also expected to be very small due to the limited phase space and the fact that this decay occurs only through the higher tail of the resonance width. PDG lists this partial decay width with respect to the $4 \pi$ partial width [68], $\Gamma(\rho \rho) / \Gamma(4 \pi) \sim 0.13$, even though these data have been omitted in extracting the resonance parameters in PDG, indicating perhaps that this measurement needs further confirmation. Using the branching ratio of $\Gamma(4 \pi) / \Gamma_{\text {total }} \sim$ 0.5 , the data lead to the partial branching ratio, $\Gamma(\rho \rho) / \Gamma_{\text {total }} \sim 0.064$. One can get an even larger branching ratio up to 0.4 if one uses the data for $\Gamma(\rho \rho) / \Gamma\left[2(\pi \pi)_{S-\text { wave }}\right]$ from Ref. [69] combined with the other ratios given in PDG. Therefore, although a consensus among the experimental data is lacking, we see that its branch ratio is not small, which can indirectly support the strong enhancement of the coupling.

Of course, our statement here needs further verification through other reaction mechanisms. One indirect way is to look into the photoproduction of double $K_{S}^{0}$ [70] through the scalar resonances $f_{0}(980)$ and $f_{0}(1500)$ where $f_{0} \rho \gamma$ or $f_{0} \omega \gamma$ vertices participate in the $t$-channel [71]. Through the vector-meson dominance, these vertices can be related to $f_{0} \rho \rho$ or $f_{0} \omega \rho$ which can be constrained by our tetraquark mixing framework.
One may ask whether there are experimental supports for the other two-vector modes with similar invariant mass, $f_{0}(1370) \rightarrow \omega \omega, f_{0}(1500) \rightarrow \omega \omega$, whose couplings are expected to enhance also in our tetraquark mixing model. Currently in PDG, there is no $\omega \omega$ mode either from $f_{0}(1370)$ or $f_{0}(1500)$. Apart from the experimental difficulty in measuring $\omega$ in comparison with $\rho$, one can understand the absence of this mode in two ways. First of all, the invariant mass of $\omega \omega$, which is $15 \mathrm{MeV}$ larger than $\rho \rho$, lies in an even higher tail of the resonance width. Also the $\omega$ decay width, $\sim 8 \mathrm{MeV}$, is much smaller than the $\rho$ decay width, $\sim 150 \mathrm{MeV}$. So the invariant mass of $\omega \omega$ should be sharp, giving it less chance to overlap with the resonance width of $f_{0}(1370)$ or $f_{0}(1500)$.

\section{SUMMARY}

In this work, we have investigated additional signatures to support the tetraquark mixing framework for the two nonets in PDG, $a_{0}(980), K_{0}^{*}(800), f_{0}(500)$, and $f_{0}(980)$ in the light nonet and $a_{0}(1450), K_{0}^{*}(1430), f_{0}(1370)$, and $f_{0}(1500)$ in the heavy nonet. In our previous works, the tetraquark mixing framework was tested through the hyperfine mass splitting, generating the experimental mass splitting between the two nonets relatively well. The predicted fall-apart decay widths into two pseudoscalar mesons are also found to be consistent with the experimental partial widths as far as the isovector resonances, $a_{0}(980)$ and $a_{0}(1450)$, are concerned. To solidify this framework, we collect more signatures to identify the two nonets as the tetraquark nonets in $\mathrm{SU}(3)_{f}$. These include the Gell-Mann-Okubo mass relation and the tetraquark mass ordering exhibited from masses of the two nonets. The marginal mass ordering seen in the heavy nonet could be another signature to support for the tetraquark mixing framework. Also the tetraquark mixing parameters are found to be independent of isospins, suggesting that the tetraquark mixing framework generates two flavor nonets in $\mathrm{SU}(3)_{f}$ which can phenomenologically match the flavor structure seen in the two nonets in PDG. As comparative models, we have examined the two-quark picture with $\ell=1$ and pointed out that its simple applications are not consistent with the two nonets phenomenologically. Alternatively the meson-meson bound picture has been discussed also with its possible limitations. We have emphasized that the fall-apart modes and their different predictions on the strength between the light and heavy nonets could be a unique feature to distinguish the tetraquark models from the mesonmeson picture and possibly from other models as well. Indeed, we have calculated the fall-apart coupling strengths into two vector mesons with interesting predictions. In particular, the coupling strengths of the two-vector modes are found to be enhanced strongly in the heavy nonet while they are suppressed in the light nonet. Their coupling ratios become huge numbers around $\sim 15$. This trend in the twovector modes can provide another testing ground for the 
tetraquark mixing framework. We have discussed some experimental hints related to the phenomena particularly from the resonances belonging to the heavy nonet.

\section{ACKNOWLEDGMENTS}

The work of H. K. and K. S. K. was supported by the National Research Foundation of Korea (NRF) funded by the Korea government (MSIT) (Grants
No. NRF-2018R1A2B6002432 and No. NRF2018R1A5A1025563). The work of M. K. C. was supported by the National Research Foundation of Korea (Grant No. NRF-2017R1E1A1A01074023). The work of D. J. was supported by a Grant-in-Aid for Scientific Research (Grant No. 17K05449) from JSPS. The work of M. O. was supported by a Grant-in-Aid for Scientific Research (Grant No. 25247036) from JSPS.
[1] S.-K. Choi et al., Bounds on the width, mass difference and other properties of $X(3872) \rightarrow \pi^{+} \pi^{-} J / \psi$ decays, Phys. Rev. D 84, 052004 (2011).

[2] R. Aaij et al. (LHCb Collaboration), Determination of the X(3872) Meson Quantum Numbers, Phys. Rev. Lett. 110, 222001 (2013).

[3] S. K. Choi et al. (Belle Collaboration), Observation of a Narrow Charmoniumlike State in Exclusive $B^{ \pm} \rightarrow$ $K^{ \pm} \pi^{+} \pi^{-} J / \psi$ Decays, Phys. Rev. Lett. 91, 262001 (2003).

[4] B. Aubert et al. (BABAR Collaboration), Search for a charged partner of the $X(3872)$ in the $B$ meson decay $B \rightarrow X^{-} K, X^{-} \rightarrow J / \psi \pi^{-} \pi^{0}$, Phys. Rev. D 71, 031501 (2005).

[5] R. Aaij et al. (LHCb Collaboration), Observation of $J / \psi \phi$ Structures Consistent with Exotic States from Amplitude Analysis of $B^{+} \rightarrow J / \psi \phi K^{+}$Decays, Phys. Rev. Lett. 118, 022003 (2017).

[6] R. Aaij et al. (LHCb Collaboration), Amplitude analysis of $B^{+} \rightarrow J / \psi \phi K^{+}$decays, Phys. Rev. D 95, 012002 (2017).

[7] V. Bhardwaj et al. (Belle Collaboration), Evidence of a New Narrow Resonance Decaying to $\chi_{c 1} \gamma$ in $B \rightarrow \chi_{c 1} \gamma K$, Phys. Rev. Lett. 111, 032001 (2013).

[8] T. Xiao, S. Dobbs, A. Tomaradze, and K. K. Seth, Observation of the charged hadron $Z_{c}^{ \pm}(3900)$ and evidence for the neutral $Z_{c}^{0}(3900)$ in $e^{+} e^{-} \rightarrow \pi \pi J / \psi$ at $\sqrt{s}=4170 \mathrm{MeV}$, Phys. Lett. B 727, 366 (2013).

[9] K. Abe et al. (Belle Collaboration), Observation of a Charmoniumlike State Produced in Association with a $J / \psi$ in $e^{+} e^{-}$Annihilation at $\sqrt{s} \approx 10.6 \mathrm{GeV}$, Phys. Rev. Lett. 98, 082001 (2007).

[10] R. Aaij et al. (LHCb Collaboration), Observation of $J / \psi p$ Resonances Consistent with Pentaquark States in $\Lambda_{b}^{0} \rightarrow$ $J / \psi K^{-} p$ Decays, Phys. Rev. Lett. 115, 072001 (2015).

[11] V. M. Abazov et al. (D0 Collaboration), Evidence for a $B_{s}^{0} \pi^{ \pm}$State, Phys. Rev. Lett. 117, 022003 (2016).

[12] L. Maiani, F. Piccinini, A. D. Polosa, and V. Riquer, Diquark-antidiquarks with hidden or open charm and the nature of $X(3872)$, Phys. Rev. D 71, 014028 (2005).

[13] H. Kim, K. S. Kim, M.-K. Cheoun, D. Jido, and M. Oka, Testing the tetraquark structure for the $X$ resonances in the low-lying region, Eur. Phys. J. A 52, 184 (2016).

[14] M. N. Anwar, J. Ferretti, and E. Santopinto, Spectroscopy of the hidden-charm $[q c][\bar{q} \bar{c}]$ and $[s c][\bar{s} \bar{c}]$ tetraquarks, Phys. Rev. D 98, 094015 (2018).
[15] L. Zhao, W. Z. Deng, and S. L. Zhu, Hidden-charm tetraquarks and charged $Z_{c}$ states, Phys. Rev. D 90, 094031 (2014).

[16] H. Kim, M.-K. Cheoun, and Y. Oh, Four-quark structure of the excited states of heavy mesons, Phys. Rev. D 91, 014021 (2015).

[17] X. Yan, B. Zhong, and R. Zhu, Doubly charmed tetraquarks in a diquark-antidiquark model, Int. J. Mod. Phys. A 33, 1850096 (2018).

[18] M. Karliner and J. L. Rosner, Discovery of DoublyCharmed $\Xi_{c c}$ Baryon Implies a Stable $(b b \bar{u} \bar{d})$ Tetraquark, Phys. Rev. Lett. 119, 202001 (2017).

[19] T. Hyodo, Y. R. Liu, M. Oka, and S. Yasui, Spectroscopy and production of doubly charmed tetraquarks, arXiv: 1708.05169.

[20] A. Esposito, M. Papinutto, A. Pilloni, A. D. Polosa, and N. Tantalo, Doubly charmed tetraquarks in $B_{c}$ and $\Xi_{b c}$ decays, Phys. Rev. D 88, 054029 (2013).

[21] E. J. Eichten and C. Quigg, Heavy-Quark Symmetry Implies Stable Heavy Tetraquark Mesons $Q_{i} Q_{j} \bar{q}_{k} \bar{q}_{l}$, Phys. Rev. Lett. 119, 202002 (2017).

[22] K. Chen, X. Liu, J. Wu, Y. R. Liu, and S. L. Zhu, Triply heavy tetraquark states with the $Q Q \bar{Q} \bar{q}$ configuration, Eur. Phys. J. A 53, 5 (2017).

[23] R. J. Lloyd and J. P. Vary, All charm tetraquarks, Phys. Rev. D 70, 014009 (2004).

[24] J. M. Richard, A. Valcarce, and J. Vijande, String dynamics and metastability of all-heavy tetraquarks, Phys. Rev. D 95, 054019 (2017).

[25] M. Karliner, S. Nussinov, and J. L. Rosner, $Q Q \bar{Q} \bar{Q}$ states: Masses, production, and decays, Phys. Rev. D 95, 034011 (2017).

[26] A. Esposito and A. D. Polosa, A $b b \bar{b} \bar{b}$ di-bottomonium at the LHC, Eur. Phys. J. C 78, 782 (2018).

[27] R. L. Jaffe, Multiquark hadrons. 1. The phenomenology of $Q \bar{Q}^{2}$ mesons, Phys. Rev. D 15, 267 (1977).

[28] R. L. Jaffe, Multiquark hadrons. 2. Methods, Phys. Rev. D 15, 281 (1977).

[29] R. L. Jaffe, Exotica, Phys. Rep. 409, 1 (2005).

[30] R. L. Jaffe, Color, spin, and flavor dependent forces in quantum chromodynamics, arXiv:hep-ph/0001123.

[31] L. Maiani, F. Piccinini, A. D. Polosa, and V. Riquer, A New Look at Scalar Mesons, Phys. Rev. Lett. 93, 212002 (2004). 
[32] D. Ebert, R. Faustov, and V. Galkin, Masses of light tetraquarks and scalar mesons in the relativistic quark model, Eur. Phys. J. C 60, 273 (2009).

[33] E. Santopinto and G. Galata, Spectroscopy of tetraquark states, Phys. Rev. C 75, 045206 (2007).

[34] S. S. Agaev, K. Azizi, and H. Sundu, The structure, mixing angle, mass and couplings of the light scalar $f_{0}(500)$ and $f_{0}(980)$ mesons, Phys. Lett. B 781, 279 (2018).

[35] S. S. Agaev, K. Azizi, and H. Sundu, The nonet of the light scalar tetraquarks: The mesons $a_{0}(980)$ and $K_{0}^{*}(800)$, arXiv: 1804.02519.

[36] H. Kim, M.-K. Cheoun, and K. S. Kim, Spin-1 diquark contributing to the formation of tetraquarks in light mesons, Eur. Phys. J. C 77, 173 (2017); Erratum, Eur. Phys. J. C77, 545(E) (2017).

[37] K. S. Kim and H. Kim, Possible signatures for tetraquarks from the decays of $a_{0}(980), a_{0}(1450)$, Eur. Phys. J. C 77, 435 (2017).

[38] H. Kim, K. S. Kim, M.-K. Cheoun, and M. Oka, Tetraquark mixing framework for isoscalar resonances in light mesons, Phys. Rev. D 97, 094005 (2018).

[39] H. Kim and Y. Oh, $D_{s}(2317)$ as a four-quark state in QCD sum rules, Phys. Rev. D 72, 074012 (2005).

[40] C. Patrignani et al. (Particle Data Group), Review of particle physics, Chin. Phys. C 40, 100001 (2016).

[41] J. J. Dudek, R. G. Edwards, and D. J. Wilson, An $a_{0}$ resonance in strongly coupled $\pi \eta, K \bar{K}$ scattering from lattice QCD, Phys. Rev. D 93, 094506 (2016).

[42] G. Janssen, B. C. Pearce, K. Holinde, and J. Speth, On the structure of the scalar mesons $f_{0}(975)$ and $a_{0}(980)$, Phys. Rev. D 52, 2690 (1995).

[43] T. Branz, T. Gutsche, and V.E. Lyubovitskij, $f_{0}(980)$ meson as a $K \bar{K}$ molecule in a phenomenological Lagrangian approach, Eur. Phys. J. A 37, 303 (2008).

[44] T. Branz, T. Gutsche, and V. E. Lyubovitskij, Strong and radiative decays of the scalars $f_{0}(980)$ and $a_{0}(980)$ in a hadronic molecule approach, Phys. Rev. D 78, 114004 (2008).

[45] F. K. Guo, C. Hanhart, U. G. Meiner, Q. Wang, Q. Zhao, and B. S. Zou, Hadronic molecules, Rev. Mod. Phys. 90, 015004 (2018).

[46] C. Hanhart, Theory concepts for heavy exotic mesons, Int. J. Mod. Phys. Conf. Ser. 46, 1860004 (2018).

[47] E. S. Swanson, Diagnostic decays of the $X(3872)$, Phys. Lett. B 598, 197 (2004).

[48] N. A. Tornqvist, Isospin breaking of the narrow charmonium state of Belle at $3872 \mathrm{MeV}$ as a deuson, Phys. Lett. B 590, 209 (2004).

[49] H.-C. Kim and M. Shmatikov, Elusive exotic states, Phys. Lett. B 375, 310 (1996).

[50] Q. Wang, Exotic candidates with heavy quark(s), Proc. Sci., Hadron2017 (2018) 144.

[51] E. van Beveren, T. A. Rijken, K. Metzger, C. Dullemond, G. Rupp, and J. E. Ribeiro, A low lying scalar meson nonet in a unitarized meson model, Z. Phys. C 30, 615 (1986).
[52] N. A. Tornqvist, Understanding the scalar meson $q \bar{q}$ nonet, Z. Phys. C 68, 647 (1995).

[53] M. Boglione and M. R. Pennington, Dynamical generation of scalar mesons, Phys. Rev. D 65, 114010 (2002).

[54] T. Wolkanowski, F. Giacosa, and D. H. Rischke, $a_{0}(980)$ revisited, Phys. Rev. D 93, 014002 (2016).

[55] L. Maiani, F. Piccinini, A. D. Polosa, and V. Riquer, Positive parity scalar mesons in the $1-2 \mathrm{GeV}$ mass range, Eur. Phys. J. C 50, 609 (2007).

[56] D. Black, A. H. Fariborz, F. Sannino, and J. Schechter, Putative light scalar nonet, Phys. Rev. D 59, 074026 (1999).

[57] D. Black, A. H. Fariborz, and J. Schechter, Mechanism for a next-to-lowest lying scalar meson nonet, Phys. Rev. D 61, 074001 (2000).

[58] A. E. Dorokhov, N. I. Kochelev, and Y. A. Zubov, Fourquark states and nucleon-antinucleon annihilation within the quark model with QCD vacuum-induced interaction, Z. Phys. C 65, 667 (1995).

[59] Y. Oh and H. Kim, Pentaquark baryons in SU(3) quark model, Phys. Rev. D 70, 094022 (2004).

[60] A. De Rujula, H. Georgi, and S. L. Glashow, Hadron masses in a gauge theory, Phys. Rev. D 12, 147 (1975).

[61] B. Keren-Zur, Testing confining potentials through meson/ baryon hyperfine splitting ratio, Ann. Phys. (Amsterdam) 323, 631 (2008).

[62] B. Silvestre-Brac, Systematics of $Q^{2}\left(\bar{Q}^{2}\right)$ systems with a chromomagnetic interaction, Phys. Rev. D 46, 2179 (1992).

[63] S. Gasiorowicz and J. L. Rosner, Hadron spectra and quarks, Am. J. Phys. 49, 954 (1981).

[64] J. Sexton, A. Vaccarino, and D. Weingarten, Numerical Evidence for the Observation of a Scalar Glueball, Phys. Rev. Lett. 75, 4563 (1995).

[65] T. Hyodo, D. Jido, and A. Hosaka, Exotic Hadrons in s-Wave Chiral Dynamics, Phys. Rev. Lett. 97, 192002 (2006).

[66] T. Hyodo, D. Jido, and A. Hosaka, Study of exotic hadrons in s-wave scatterings induced by chiral interaction in the flavor symmetric limit, Phys. Rev. D 75, 034002 (2007).

[67] S. H. Lee, H. Kim, and Y. Oh, Decay modes of ideally mixed narrow pentaquark states, J. Korean Phys. Soc. 46, 774 (2005).

[68] A. Abele et al. (CRYSTAL BARREL Collaboration), $4 \pi$ decays of scalar and vector mesons, Eur. Phys. J. C 21, 261 (2001).

[69] D. Barberis et al. (WA102 Collaboration), A spin analysis of the $4 \pi$ channels produced in central $p p$ interactions at $450 \mathrm{GeV} / \mathrm{c}$, Phys. Lett. B 471, 440 (2000).

[70] S. Chandavar et al. (CLAS Collaboration), Double $K_{S}^{0}$ photoproduction off the proton at CLAS, Phys. Rev. C 97, 025203 (2018).

[71] H. Xing, C. S. An, J. J. Xie, and G. Li, Photoproduction of $f_{0}(980)$ and $f_{0}(1500)$ resonances off a proton target, Phys. Rev. D 98, 094007 (2018). 